Review

\title{
MiRNAs in Radiotherapy Resistance of Nasopharyngeal Carcinoma
}

Yutong Tian ${ }^{1,2}$, Lu Tang ${ }^{1,2}$, Pin Yij ${ }^{1,2}$, Qing Pan ${ }^{1,2}$, Yaqian Han ${ }^{1}$, Yingrui Shi ${ }^{1}$, Shan Rao $^{1}$, Shiming Tan ${ }^{1}$, Longzheng Xia ${ }^{1}$, Jinguan Lin ${ }^{1}$, Linda Oyang ${ }^{1}$, Yanyan Tang ${ }^{1}$, Jiaxin Liang ${ }^{1}$, Xia Luo ${ }^{1}$, Qianjin Liao ${ }^{1}$, Hui Wang $^{1 凶}$, Yujuan Zhou ${ }^{\circledR}$

1. The Affiliated Cancer Hospital of Xiangya School of Medicine, Central South University and Hunan Cancer Hospital, Key Laboratory of Translational Radiation Oncology, Hunan Province, 283 Tongzipo Road, Changsha 410013, Hunan, China.

2. University of South China, Hengyang, 421001, Hunan, China.

$\triangle$ Corresponding author: Yujuan Zhou or Hui Wang, Hunan Key Laboratory of Translational Radiation Oncology, Hunan Cancer Hospital and The Affiliated Cancer Hospital of Xiangya School of Medicine, Central South University, 283 Tongzipo Road, Changsha 410013, Hunan, China. Tel: 86-731-88651681; Fax: 86-731-88651999; Email: yujany_zhou@163.com or, Email: wanghui710327@163.com.

(c) The author(s). This is an open access article distributed under the terms of the Creative Commons Attribution License (https://creativecommons.org/licenses/by/4.0/). See http://ivyspring.com/terms for full terms and conditions.

Received: 2019.12.04; Accepted: 2020.03.27; Published: 2020.04.06

\begin{abstract}
Nasopharyngeal carcinoma (NPC) is one of the most common malignant tumors of the head and neck in Southeast Asia and southern China. Although the comprehensive treatment based on intensity-modulated radiation therapy improves outcomes, the five-year survival rate of NPC patients is low, and the recurrence remains high. Radiotherapy resistance is the main cause of poor prognosis in NPC patients. MicroRNAs (miRNAs) are a class of endogenous non-coding RNAs regulating various biological functions in eukaryotes. These miRNAs can regulate the development and progression of nasopharyngeal carcinoma by affecting the proliferation, apoptosis, movement, invasion and metastasis of NPC cells. The abnormal expression of miRNAs is closely related to radiotherapy sensitivity and prognosis of NPC patients, which can affect the transmission of related signaling pathways by regulating the expression of tumor suppressor genes and / or oncogenes, and therefore participate in radiotherapy resistance in nasopharyngeal carcinoma. Here, we review the mechanisms by which miRNAs may be involved in the radiotherapy resistance of nasopharyngeal carcinoma.
\end{abstract}

Key words: nasopharyngeal carcinoma; radiotherapy resistance; miRNAs; apoptosis; miR-BARTs

\section{Introduction}

Nasopharyngeal carcinoma (NPC) is a malignant tumor originating from nasopharyngeal epithelial cells. Etiological factors include genetics, EBV infection, environment, smoking and other factors [1, 2]. Nasopharyngeal carcinoma occurs mainly in Southeast Asia and southern China, and its morbidity and mortality rank first in head and neck malignant tumors [3-5]. NPC is mainly poorly differentiated squamous cell carcinoma and undifferentiated carcinoma; the special anatomy and local invasive growth characteristics of NPC make it unsuitable for surgical treatment, and radiotherapy is the best choice for NPC. Although the radiotherapy is more advanced, the recurrence rate of nasopharyngeal carcinoma is still high at $82 \%$. Radiotherapy resistance is the leading cause of local recurrence and distant metastasis [6, 7].

MicroRNAs (miRNAs) are a type of endogenous non-coding RNA with a length of about $22 \mathrm{nt}$ (19-25 $\mathrm{nt})$. They bind to target mRNA molecules through partial or complete complementary sequences or to specific proteins to form miRNA-induced silencing complex and inhibit the translation of target mRNAs [8]. Many previous studies have found that miRNAs regulate the expression of key genes in cell cycle, apoptosis and migration, and affect the proliferation, invasion and metastasis of various tumor cells including nasopharyngeal carcinoma [9-14]. Some 
scholars have compared microarray data of miRNA expression profiles in radiotherapy-sensitive and radiation-resistant nasopharyngeal carcinoma cells, identified differentially expressed miRNAs and mRNAs, and constructed a post-transcriptional regulatory network of more than 300 miRNA target gene pairs $[11,15]$. Similar studies have been reported by other scholars to show that miRNAs ectopically expressed in nasopharyngeal carcinoma cells affects the expression of their target genes or proteins, thereby affecting the relevant signaling pathways and thereby participating in radiotherapy for nasopharyngeal carcinoma [16]. This article updates the differential expression of miRNA molecules in the radiotherapy resistance of nasopharyngeal carcinoma, and explores the mechanism of miRNA in the radiotherapy resistance of nasopharyngeal carcinoma.

\section{Mechanism of radiotherapy resistance in nasopharyngeal carcinoma}

Radiation therapy (RT) is a local treatment for curing or palliative treatment of tumors. RT works through damaging the DNA strands of tumor cells to directly kill tumor cells or make them lose the ability of infinite proliferative. However, increased tumor volume, oxygen reduction, and dysregulation of various genes can lead to tolerance of tumor cells to radiation, and thus reduce sensitivity, i.e., radiotherapy resistance. The specificity of radiotherapy resistance is the decrease of apoptosis after irradiation. The reasons at the gene level include the following two types: the decrease of apoptotic genes or the increase of the expression of anti-apoptotic genes and proliferating genes, and the enhancement of genes related to DNA damage repair, or the expression of cell cycle regulatory genes is dysregulated $[6,7,17,18]$. Many studies have shown that miRNAs participate in tumor radiation resistance through the above mechanisms [19-21].

Apoptosis is the most important mechanism of radiation therapy [22]. Abnormal expression of apoptosis-related genes can inhibit the apoptosis of tumor cells induced by ionizing radiation and increase their survival, thereby promoting the radiotherapy resistance of NPC. The role of genes regulating cell proliferation is opposite to that of apoptotic genes, which can promote the survival and proliferation of tumor cells. The abnormal activation and expression of cell proliferation genes is also one of the mechanisms of tumor radiotherapy resistance. There are a large number of genes involved in proliferation and apoptosis, which are mainly divided into the following families: caspase, bcl-2, fas, and the most frequently mutated p53 gene in tumors. Proliferation-related genes are mainly myc, some factors in the bcl-2 family, and so on. They participate in the regulation of apoptosis, either alone or in concert [23-26]. Mutations in the p53 gene occur in nearly $50 \%$ of human malignancies, which greatly increases the cell resistance to radiation [27, 28]. Studies have shown that up-regulation of Bcl-2 expression can inhibit the expression of Bax, caspase-3 and other apoptotic genes and promotes the survival of nasopharyngeal carcinoma cells, thus playing a role in the development and metastasis of nasopharyngeal carcinoma [29]. Fas belongs to the TNF receptor family. Usually, when fas protein binds to its ligand, it activates caspases, which causes apoptosis of targeted cells.

Radiation directly ionizes target cell DNA or ionize water to generate free radicals that cause DNA damage in target cells, including double strained break (DSB) and single strained break (SSB). DSB mainly causes irreversible fatal injury. SSB mainly causes reversible potential lethal damage (PLD) and sub-lethal damage (SLD) under certain conditions. The hallmark of DNA double-strand break (DSB) is phosphorylation of histone $\mathrm{H} 2 \mathrm{AX}$ in chromatin to produce $\gamma-\mathrm{H} 2 \mathrm{AX}$ [30]. In eukaryotes, DNA damage is repaired after irradiation through DNA damage response (DDR) [31]. The repair of DNA damage greatly affects the survival of tumor cells, producing the resistance of radiotherapy.

Experiments have confirmed that the radiosensitivity of tumor cells is closely related to the distribution of tumor cell cycle phases. At different phases of the cell cycle, the ability of radiation to kill cells is different. The most sensitive to radiation in the cell cycle is the M phase, followed by the G2 phase, and the cells in the G1 and S phases are less sensitive to radiation. Because of the active DNA segregation and cell division activity in G2 and $M$ phases, radiation can easily cause DNA damage and cell damage, leading to unprogrammed cell death. Moreover, the periodic checkpoint is also an important part of DNA damage repair. The tumor suppressor gene p53 removes DNA abnormalities through cell cycle inhibition and induction of apoptosis.

\section{MiRNAs affect the sensitivity of radiotherapy for nasopharyngeal carcinoma}

Since miRNA was discovered in Caenorhabditis elegans, its role as a non-coding RNA in regulating various biological behaviors of animals and plants has received widespread attention, especially in oncology [32]. MiRNAs have been widely reported to regulate the occurrence and development of tumors by binding to their targeted 
mRNAs or by interacting with other non-coding RNAs, and this is not an exception in nasopharyngeal carcinoma. Many miRNAs have been found to be differentially expressed between patients with nasopharyngeal carcinoma and normal people [33, 34]. These miRNAs can be roughly divided into two types; one is encoded by human cells and the other is Epstein-Barr virus (EBV) [35]. These miRNAs affect the proliferation, invasion and migration, apoptosis, therapeutic sensitivity and other biological behaviors of NPC [36-38].

Radiotherapy sensitivity of tumors is an important basis for tumor radiotherapy. Several groups of researchers have identified differentially expressed miRNAs in radiosensitivity and radiation-resistant cells of nasopharyngeal carcinoma by gene sequencing and microarray analysis. miRNA-762, miRNA-1202, is up-regulated in radiotherapy-resistant nasopharyngeal carcinoma cells. miRNA-4291, miR-19b-3p, miR-21, miRNA-23a, miR-205, miR-483, let-7e, miR-193b and miR-7, etc., more than 60 , can regulate nasopharyngeal carcinoma radiation resistance. The down-regulated miRNAs includes miR-125, miR-660, miR-203, miR-130a, miR-30a and miR-23a, more than 30, which can regulate the sensitivity of radiotherapy for nasopharyngeal carcinoma. These up-regulated or down-regulated miRNAs can regulate the radiotherapy resistance of nasopharyngeal carcinoma cells by acting on different target genes and pathways [16, 39, 40]. For example, miR-205 inhibits PTEN, thereby promoting AKT to increase the resistance of nasopharyngeal carcinoma cells to radiation, facilitating their survival after irradiation [41, 42]. MiR-20a-5p reduces the sensitivity of nasopharyngeal carcinoma cells to radiotherapy by targeting NPAS2 and Rab27B, and miR-193a-3p enhances the anti-radiation ability of NPC by targeting SRSF2 [43-45]. Different miRNAs can directly reduce the sensitivity of nasopharyngeal carcinoma cells to radiation by directly or indirectly regulating different signaling pathways, but the expression and up-regulation of miRNAs that enhance radiosensitivity in nasopharyngeal carcinoma are rarely reported.

\section{Mechanisms of miRNAs in radiotherapy resistance in nasopharyngeal carcinoma}

In this section, we mainly describe the molecular mechanisms of miRNA involved in the radiotherapy resistance of nasopharyngeal carcinoma by affecting apoptosis, DNA damage repair and cell cycle progression of nasopharyngeal carcinoma cells.
MiRNAs participate in radiotherapy resistance of nasopharyngeal carcinoma by affecting the transmission of bcl-2 apoptosis family, Caspase and Fas family signals

MiR-19b-3p, miR-125b, miR-21, and miR-205 promote the radiotherapy resistance of nasopharyngeal carcinoma by regulating the Bcl-2 gene family protein [6]. miR-185 regulates the $\mathrm{Bcl}-2$ family to promote the sensitivity of nasopharyngeal carcinoma cells to radiation. Bcl-2 is an apoptosis-inhibiting gene, and at least 19 homologs have been discovered, which play a regulatory role in the mitochondrial-dependent apoptotic pathway and control the release of cytochrome $\mathrm{c}$ and other apoptotic factors in mitochondria [46, 47]. Members of the Bcl-2 family contain 1-4 Bcl-2 homology domains (BH1-4), of which $\mathrm{BH} 4$ is a domain unique to anti-apoptotic proteins, and $\mathrm{BH} 3$ is a domain involved in promoting apoptosis. According to function and structure, the Bcl-2 gene family can be divided into two groups; one is anti-apoptosis, such as Bcl-2, Bcl-xl, Bcl-w, and Mcl-1; the other is pro-apoptosis, including: Bax, Bad, Bak, Bid, Bim, Puma, etc. NF-кB is a transcription factor of Bcl-2, which can induce the expression of anti-apoptotic genes and stimulate cell proliferation and prevent apoptosis [48]. In addition, NF-KB stimulates the caspase- 8 inhibitor FLIP, an inhibitor of the apoptotic proteins c-IAP1 / 2 and XIAP.

Huang, T. et al. analyzed the expression of miR-19b-3p in five nasopharyngeal carcinoma cell lines (CNE1, CNE2, 5-8F, 6-10B, and HNE1) and normal nasopharyngeal epithelia cell line NP69 by qRT-PCR and other techniques. The expression of miR-19b-3p in radiotherapy-resistant cells of nasopharyngeal carcinoma was found to be significantly higher than that in normal nasopharyngeal tissues. Moreover, miR-19b-3p can increase the expression of $\mathrm{Bcl}-2$ by targeting tumor necrosis factor alpha-induced protein 3 (TNFAIP3, also known as A20), while the expression of apoptosis-promoting factor Bax is decreased in NPC tissues and cells. TNFAIP3 can increase apoptosis by inhibiting the expression of Bcl-2 transcription factor NF-KB. miR-19b-3p suppresses TNFAIP3 expression and activates NF-kB pathway, which reduces apoptosis of irradiated NPC cells. Similarly, Li, et al. found that miR-125b is up-regulated in nasopharyngeal carcinoma cell lines and promotes radiotherapy resistance of nasopharyngeal carcinoma cells by targeting the TNFAIP3/NF-KB pathway. However, miR-21 and miR-205 are highly expressed in NPC cells resistant to radiotherapy, and the radiotherapy resistance is associated with inhibition of PTEN signaling pathway [41, 42, 49]. Inactivation of 
PTEN results in constitutive activation of the STAT3 and PI3K-AKT pathways and subsequent protein synthesis, inhibiting apoptosis. Experiments have shown that Bcl-2 protein levels are increased in miR-205 transfected CNE-2 cells, and studies have shown that STAT3 activation can inhibit cell apoptosis by blocking the expression of Bim protein [50, 51]. However, miR-185, which enhances apoptosis, can increase the expression of Bax and Bid and decrease the expression of Bcl-2, promoting apoptosis, [52]. All these indicate that miR-19b-3p, miR-125b, miR-21, and miR-205, which are elevated in nasopharyngeal carcinoma, promote radiotherapy resistance through the bcl-2 apoptosis family, while miR-185 causes nasopharyngeal cancer cells sensitive to radiation therapy.

The researchers found that up-regulated miR-21 and miR-BART4 or down-regulated miR-143 and miR-153 in nasopharyngeal carcinoma can inhibit the apoptosis of nasopharyngeal carcinoma cells via Fas or Caspase pathway, accompanied by or not with the regulation of Bcl-2 family proteins, and the sensitivity of nasopharyngeal carcinoma cells to radiotherapy is reduced. $\mathrm{Wu}, \mathrm{Q}$. et al. found that EBV-encoded miR-BART4 promotes proliferation and invasion of nasopharyngeal carcinoma cells in nasopharyngeal carcinoma, and promotes radioresistance of nasopharyngeal carcinoma. They confirmed by further experiments that overexpression of miR-BART4 can reduce the expression of cleaved caspase-3, inhibiting apoptosis of nasopharyngeal carcinoma cells. After 6Gy irradiation treatment, the level of caspase- 3 and apoptosis in the miR-BART4 overexpressed group was significantly lower than that in the NC group and the miR-BART4 inhibitor group, and in this process, bcl-2 and bax also appeared according change [53]. Chen, J. H. and other scholars have found that miR-143, which is down-regulated in nasopharyngeal carcinoma, can initiate apoptosis through caspase-3. As the amount of transfected miR-143 increases, the expression level of caspase-3 increase aacordingly. The level of bcl-2 did not change during this process [54]. Similarly, miR-153 expression was significantly reduced in NPC patients compared to adjacent tissues. Up-regulation of miR-153 induces apoptosis, increases caspase-3 and caspase- 9 activity, and increases B-cell lymphoma 2 (Bcl-2)-associated $X$ protein/Bcl-2 protein expression in 13-9B cells [55].

It is well known that Fas, also known as APO-1, TNFR (tumor necrosis factor receptor) and the NGF receptor family can drive cells to pass the Caspase pathway when they bind to their receptors [56]. Caspases belong to cysteine proteases and are a key enzyme that causes apoptosis. Once activated, it can degrade proteins in cells and induce apoptosis. Top caspase initiation and effector caspases trigger apoptosis, and different members of the caspase family are involved in endogenous or exogenous apoptosis programs, such as caspase- 9 or caspase-10, which are involved in exogenous apoptosis $[57,58]$. Moreover, caspases play an important role in the immune system triggered by DNA virus or RNA virus infection. What role does caspase then play in nasopharyngeal carcinoma closely related to Epstein-Barr virus? Interactions that regulate tumor apoptosis are a question worthy of exploring. In addition, the replication and high expression of EBV virus is one of the important factors in the poor prognosis of nasopharyngeal carcinoma. The level of EBV DNA is closely related to local recurrence and distant metastasis after treatment in patients with nasopharyngeal carcinoma [31, 59]. EBV infection can induce the expression of a variety of miRNAs including microRNA-21, and scholars have found that Epstein-Barr virus-encoded LMP1 (latent membrane protein 1) can increase miR-21, negatively regulate pro-apoptotic factor procedural cell death 4 (PDCD4) and Fas ligand (Fas-L) increase resistance to cisplatin treatment in nasopharyngeal carcinoma cells [60]. Platinum drugs combined with radiotherapy can more effectively kill hypoxic tumor cells in less solid tumors. Based on this principle and clinical practice, platinum-based synchronous chemotherapy combined with radiotherapy is defined as the standard treatment of advanced nasopharyngeal carcinoma. This indicates that the high expression of miR-21 also indirectly causes radiotherapy resistance of nasopharyngeal carcinoma [61,62].

Among them, the genes up-regulated include miR-19b-3p, miR-125b, miR-21, miR-205, miR-BART4, miR-143, and the down-regulated genes are miR-185, miR-153.

\section{2 miRNAs participate in radiotherapy resistance of nasopharyngeal carcinoma by regulating DNA damage repair}

Although irradiated cells can be apoptotic, not all cells enter apoptosis, which is one of the causes of radiotherapy resistance. When the cells are irradiated with a non-lethal dose and the single-strand or double-strand DNA break damage is repaired, the cells may survive without apoptosis.

Zhou, X. et al. found that EBV-miR-BART8-3p promotes NPC cell proliferation after irradiation in vitro and is associated with induction of cell cycle arrest in G2/M phase. In addition, in vitro animal experiments also indicate that EBV-miR- BART8-3p significantly increased the size of xenografts in nude mice and promotes radioresistance of NPC by 
regulating the activity of the ATM/ATR signaling pathway [63]. Studies have shown that the other four miRNAs encoded by EBV (BART5-5p, BART7-3p, BART9-3p and BART14-3p) directly target endogenous ATM, and the reduced ATM also helps maintain the virus incubation period [31, 64]. MiR-24 expression was found to be decreased in nasopharyngeal carcinoma cells and patients with higher stage nasopharyngeal carcinoma, and this change was associated with the development of radiotherapy resistance $[65,66]$. Scholars also found that miR-24 can inhibit the repair of DNA damage by targeting Jab1, and thus increase the sensitivity of nasopharyngeal carcinoma cells to radiotherapy [66]. JAB1 (c-Jun activation domain binding protein-1) is involved in the repair of DNA double-strand breaks, which maintain the stability of the genome. The deletion of Jab1 leads to spontaneous DNA fragmentation and increased expression of histone $\gamma-\mathrm{H} 2 \mathrm{AX}$ [67-69].

The protein encoded by the ATM gene belongs to the PI3 / PI4 kinase family. This protein is an important cell cycle checkpoint kinase that acts as a regulator of a variety of downstream proteins, including the tumor suppressor proteins p53 and BRCA1, checkpoint kinase CHK2, checkpoint proteins RAD17 and RAD9, and the DNA repair protein NBS1. ATM participated in the cell cycle and DNA damage repair, two important processes in radiation injury $[70,71]$. In cells with mild DNA damage, ATM activates cell cycle checkpoints, stops cell cycle progression, and initiates DNA repair process. However, in cells with irreparable severe DNA damage, ATM triggers apoptosis to remove cells [72]. Moreover, some scholars have found that severe hypoxia in solid tumors increases the expression of ATM and initiates DNA repair $[64,73]$.

\section{miRNAs participate in radiotherapy resistance of nasopharyngeal carcinoma by regulating cell cycle}

In addition to DNA damage repair and apoptosis involved in nasopharyngeal carcinoma radiotherapy resistance, miRNAs also triggers the radiotherapy resistance of nasopharyngeal carcinoma by regulating the cell cycle. The cell cycle regulatory system can be easily divided into three parts, cyclins, cyclin-dependent cellular kinases (CDK), and CDK kinase inhibitors (CDKI). In mammalian cells, retinoblastoma protein $(\mathrm{Rb})$ regulates the progression of G1 to S phase [74]. At the G1 phase of the cell cycle, $\mathrm{Rb}$ binds to E2F-DP1 and inhibits downstream transcription. When the cells enter the $S$ phase, G1/S CDK inactivates $R b$, resulting in the release of $R b$ from E2F-DP1 and activation of the E2F target gene, thereby promoting G1 activation and transition to $S$ phase. Because cells in different phases have different sensitivity to radiation, cell cycle distributions directly affect the radiosensitivity of nasopharyngeal carcinoma cells.

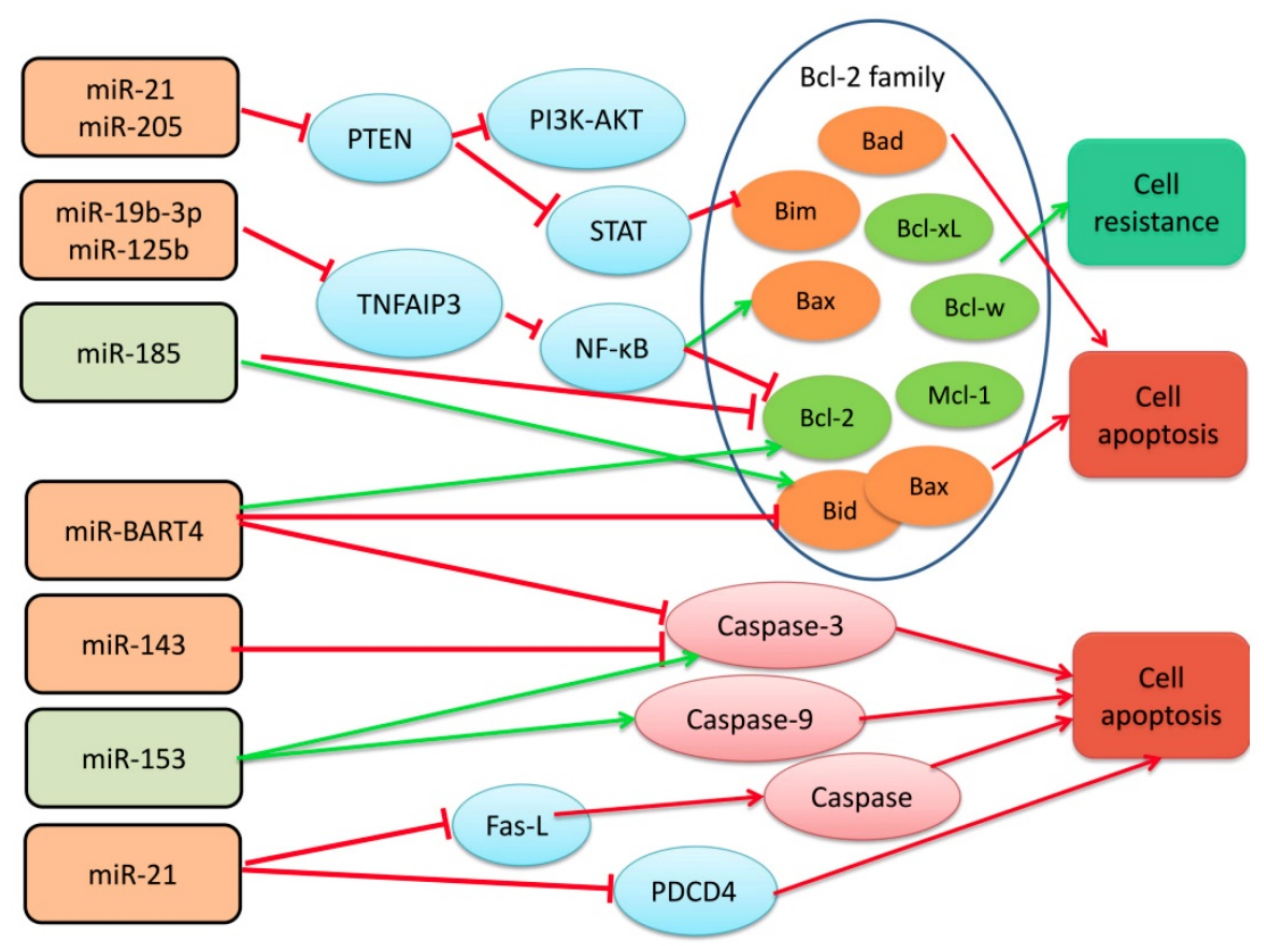

Figure 1. Schematic diagram of miRNAs that regulate radiotherapy resistance by Bcl-2, Caspase and Fas apoptosis families in nasopharyngeal carcinoma. 
$\mathrm{Wu}$, et al. found that overexpression of miR-188 blocks nasopharyngeal cancer cells in the G1 phase, through inhibiting $\mathrm{Rb}$ phosphorylation and down-regulates E2F transcription, and then inhibiting the mRNA and protein expression of CDK4 and CDK2, therefore enhances the radiosensitivity of nasopharyngeal carcinoma cells[75]. Juan $\mathrm{Lu}$ et al found that miR-26a, which is down-regulated in nasopharyngeal carcinoma cells and patients, inhibits the expression of cyclin D3, CDK4 and CDK6, but activates p14 (ARF) and p21 (CIP1) in an EZH2 (enhancer of zeste 2 polycomb repressive complex 2 subunit)-dependent manner, thus induces cell cycle arrest in G1 phase and enhances radiosensitivity of nasopharyngeal carcinoma[76]. In addition, some scholars have found that miR-23a can make more cells in the G2-M phase, indicating that miR-23a is blocked by irradiation, and the tumor cells are not sensitive to radiation [77]. In addition, serum miR-663 levels in NPC patients are significantly elevated and directly target cyclin-dependent kinase inhibitor 2A (CDKN2A), thereby promoting cell cycle progression and cell proliferation [78].

\section{miRNAs participate in radiotherapy resistance of nasopharyngeal carcinoma by other means.}

Numerous studies have shown that radiation can directly induce the production of a variety of inflammatory factors including IL-6, IL-8, IL-1a, IL-1 $\beta$, IL-18, TNF- $\alpha$, IFN- $\gamma$, etc. Inflammation, the tumor microenvironment of pro-oxidants, eventually leads to tumor cell death $[79,80]$. MiRNAs target these inflammatory factors to participate in the radiosensitivity of nasopharyngeal carcinoma. Studies have shown that both down-regulated miR-23a and miR-203 in nasopharyngeal carcinoma radiotherapy-resistant cell lines (CNE-2-IR) can target IL8 and activate Stat3, which inhibits apoptosis, or AKT that affects radiotherapy resistance in nasopharyngeal carcinoma [77, 81]. Blood IL-17 was significantly elevated in NPC patients compared with normal subjects. The expression of miR-135a in cancer cells isolated from nasopharyngeal tumors was significantly lower than in NP69 cells, and inhibition of IL-17 by miR-135a mimics resulted in significant inhibition of NPC cell proliferation. These findings suggest that down-regulation of miR-135a may contribute to the development of NPC through a mechanism by which IL-17 stimulates the expression of pro-inflammatory cytokines [82]. Moreover, the aforementioned NF- $\mathrm{kB}$ signaling pathway is also involved in the release and regulation of inflammatory molecules. In addition, IL-6 molecules have been reported to promote Stat 3 and Nrf2-antioxidant pathway in oral squamous cell carcinoma, and up-regulation of Mn-SOD reduces oxidative stress leading to radiotherapy resistance [83]. However, more miRNAs and inflammatory factors play a role in radiotherapy resistance and specific pathways remain to be further explored.

Table 1. miRNAs involved in NPC radioresistance

\begin{tabular}{|c|c|c|c|c|c|}
\hline miRNA & Expression & Source & Targets or Pathway & Function & References \\
\hline miR-19b-3p & up & cell and tissue & $\begin{array}{l}\text { Bcl-2,Bax, Capase3, c-Capase3, } \\
\text { TNFAIP3(A20)/ NF-кB }\end{array}$ & cell apoptosis $\downarrow$ & [6] \\
\hline miR-125b & up & tissue & $\begin{array}{l}\text { p-p65, } \mathrm{\gamma}-\mathrm{H} 2 \mathrm{AX} \\
\text { TNFAIP3 (A20)/ NF-kB }\end{array}$ & cell apoptosis $\downarrow$ & [29] \\
\hline miR-21 & up & cell and tissue & $\begin{array}{l}\text { Bcl-2, Bax, } \\
\text { PTEN/AKT }\end{array}$ & $\begin{array}{l}\text { cell survival } \uparrow, \\
\text { cell apoptosis } \downarrow\end{array}$ & {$[49,91]$} \\
\hline miR-205 & up & cell & Bcl-2, Bax, snail, PTEN/AKT & $\begin{array}{l}\text { cell survival } \uparrow, \\
\text { cell apoptosis } \downarrow\end{array}$ & [41] \\
\hline $\begin{array}{l}\text { EBV-miR-BART8-3p, BART5-5p, } \\
\text { BART7-3p, BART9-3p, BART14-3p }\end{array}$ & up & cell and tissue & $\gamma$-H2AX, ATM & $\begin{array}{l}\mathrm{DDR} \uparrow, \\
\text { cell apoptosis } \downarrow\end{array}$ & [31] $[64,72]$ \\
\hline $\operatorname{miR-23a}$ & down & cell and tissue & $\gamma \mathrm{H} 2 \mathrm{AX}, \mathrm{IL}-8$ / Stat3 & $\begin{array}{l}\text { DDR } \downarrow \text {, } \\
\text { cell cycle } \downarrow \text {, } \\
\text { cell apoptosis } \uparrow\end{array}$ & [77] \\
\hline miR-203 & down & tissue & $\gamma \mathrm{H} 2 \mathrm{AX}, \mathrm{IL}-8$ / Stat3 & $\begin{array}{l}\text { DDR } \downarrow \text {, } \\
\text { cell cycle } \downarrow \text {, } \\
\text { cell apoptosis } \uparrow\end{array}$ & [81] \\
\hline miR-20a-5p & up & cell & $\gamma \mathrm{H} 2 \mathrm{AX}, \mathrm{Rab} 27 \mathrm{~B}, \mathrm{NPAS} 2$ & $\begin{array}{l}\text { cell survival } \uparrow, \\
\text { cell apoptosis } \downarrow\end{array}$ & {$[43,44]$} \\
\hline miR-193a-3p & up & cell & SRSF2 & cell cycle $\downarrow$ & [45] \\
\hline $\operatorname{miR}-24$ & down & tissue & SP1 & $\mathrm{DDR} \downarrow$ & [65] \\
\hline miR-26a & down & cell and tissue & CDK4,CDK6, EZH2 & cell cycle $\downarrow$ & [76] \\
\hline $\operatorname{miR}-135 a$ & down & cell & IL-17 & $\begin{array}{l}\text { inflammation } \downarrow \text {, } \\
\text { cell apoptosis } \uparrow\end{array}$ & [82] \\
\hline miR-143 & down & cell and tissues & capase3,PARP, $\gamma \mathrm{H} 2 \mathrm{AX}$ & $\mathrm{DDR} \downarrow$ & [87] \\
\hline
\end{tabular}

' $\uparrow$ ' and ' $\downarrow$ ' stand for the miRNA inhibiting or promoting the function, respectively. 
The stemness of tumor cells plays an important role in the growth, metastasis and drug resistance of various tumors [84]. MiRNAs are also involved in the maintenance and enhancement of the stemness of tumor cells. Previous studies have found that a variety of miRNAs were involved in the maintenance of the stemness of nasopharyngeal carcinoma tumor cells, leading to tumor progression and therapy resistance. For example, Cai, et al. found that miR-BART7-3p encoded by EBV can increase the number of side population (SP) cells in nasopharyngeal carcinoma cells compared with control cells by targeting SMAD7, while the SP cells are reported to be primitive stem-like population[85]. Wang, et al. found that c-MYB strengthens the stemness of NPC cancer stem cell via down-regulating miR-143, thereby increasing the resistance to radiotherapy [86]. Other articles also mentioned the effects of miRNAs on the stemness of nasopharyngeal carcinoma cells and the regulation of apoptosis and damage repair of cells by affecting stemness [87].

Local recurrence and metastasis after intensive radiotherapy are the main manifestations of radiotherapy failure. Studies have shown that miR-BART7 and miR-BART13 encoded by EBV and Epstein-Barr virus (EBV) immunoglobulin A / capsid antigen (IgA / VCA) and antigen (IgA / EA) have similar effects in predicting the recurrence and metastasis of nasopharyngeal carcinoma [88]. Radiotherapy can significantly reduce miR-BART7 and miR-BART13 in the plasma of patients with nasopharyngeal carcinoma, but those patients with signs of local or regional persistent tumors or metastases have no significant decrease after radiotherapy [88]. Other scholars have found that miR-150 expression is elevated in patients with nasopharyngeal carcinoma, and that the high expression of miR-150 is significantly related to tumor recurrence after radiotherapy; the target molecule FoxO4 of miR-150 is significantly reduced during this process [89]. Thus miR-150 and its target molecule FoxO4 may be a predictors of nasopharyngeal carcinoma recurrence. In addition, various miRNAs have been reported as plasma biomarkers for monitoring the recurrence and metastasis of nasopharyngeal carcinoma; the abnormal expression of these miRNAs in nasopharyngeal carcinoma affects the sensitivity of nasopharyngeal carcinoma to radiotherapy and chemotherapy, leading to the recurrence and metastasis of nasopharyngeal carcinoma and ultimately to poor patient prognosis $[35,90]$. However, their specific mechanism needs further research and exploration.

\section{Prospect}

Radiotherapy resistance of nasopharyngeal carcinoma causes poor local control, recurrence and distant metastasis, which is significantly associated with poor prognosis. In recent years, miRNAs have been found to be involved in the regulation of tumor development and progression by regulating target gene expression. In miRNAs associated with radiation resistance in nasopharyngeal carcinoma, up- or down-regulations of miRNAs have been found to be closely related to radioresistance. In addition to the miRNA molecules discussed above, there are still many miRNA molecules involved in radiotherapy resistance of nasopharyngeal carcinoma, such as miR-203, miR-150, miR-324-3p, miR-222, and miR-495, etc. The mechanisms of regulating the radiotherapy resistance of nasopharyngeal carcinoma by miRNAs can be roughly classified into the above-mentioned regulation of apoptosis cell cycle or DNA damage repair, but whether they function alone or jointly to regulate the nasopharyngeal radiotherapy resistance is not clear yet [81, 92-95].

By detecting the expression level of specific miRNA in tissues or serum of patients with nasopharyngeal carcinoma, the radiotherapy effect may be predicted, and the nasopharyngeal carcinoma tissues and cells are sensitive to radiotherapy by changing the content of these specific miRNAs at the pre-transcriptional level. According to its regulation of the body immunity, combined with immunotherapy, the therapeutic effect of the tumor is further enhanced so that the tumor patients get the maximal benefit [96, 97]. However, miRNAs are involved directly or indirectly in the regulation of multiple pathways, and are found to play an opposite role in many tumors. The mechanism is still unclear and further research is needed. Moreover, whether there are other miRNAs involved in the regulation of radiotherapy resistance of nasopharyngeal carcinoma and whether other mechanisms and pathways are involved, extensive research and discovery are still needed.

\section{Acknowledgements}

This work was supported in part by grants from the following sources: the National Natural Science Foundation of China (81972636, 81872281), the Natural Science Foundation of Hunan Province (2019JJ40175, 2019JJ40183, 2018JJ1013, 2017JJ3190), the Research Project of the Health and Family Planning Commission of Hunan Province (B20180400, B20180582), and the Changsha Science and Technology Board (kq1706045, kq1706043), Ascend Foundation of National cancer center (NCC2018b68). 


\section{Competing Interests}

The authors have declared that no competing interest exists.

\section{References}

1. Lu Y, Ma J, Li Y, Huang J, Zhang S, Yin Z, et al. CDP138 silencing inhibits TGF- $\beta /$ Smad signaling to impair radioresistance and metastasis via GDF15 in lung cancer. Cell Death \& Disease. 2017; 8(9): e3036.

2. Tu C, Zeng Z, Qi P, Li X, Yu Z, Guo C, et al. Genome-Wide Analysis of 18 Epstein-Barr Viruses Isolated from Primary Nasopharyngeal Carcinoma Biopsy Specimens. Journal of Virology. 2017; 91(17): e00301-17.

3. Jiang N, Jiang $X$, Chen Z, Song $X, W u$ L, Zong D, et al. MiR-203a-3p suppresses cell proliferation and metastasis through inhibiting LASP1 in nasopharyngeal carcinoma. Journal of experimental \& clinical cancer research. 2017; 36(1): 138.

4. Long $\mathrm{M}, \mathrm{Fu} \mathrm{Z}, \mathrm{Li} \mathrm{P}, \mathrm{Nie} \mathrm{Z}$. Cigarette smoking and the risk of nasopharyngeal carcinoma: a meta-analysis of epidemiological studies. BMJ Open. 2017; 7(10): e016582.

5. Chen W, Zheng R, Zhang S, Zeng H, Xia C, Zuo T, et al. Cancer incidence and mortality in China, 2013. Cancer Lett. 2017; 401: 63-71.

6. Teng H, Li Y, Jing W, Gu JJ, Wu JZ, Dan C, et al. MicroRNA-19b-3p regulates nasopharyngeal carcinoma radiosensitivity by targeting TNFAIP3/NF-kB axis. Journal of Experimental \& Clinical Cancer Research. 2016; 35(1): 188.

7. Wang S, Zhang R, Claret FX, Yang H. Involvement of microRNA-24 and DNA methylation in resistance of nasopharyngeal carcinoma to ionizing radiation. Molecular Cancer Therapeutics. 2014; 13(12): 3163-74.

8. Fabian MR, Sonenberg N. The mechanics of miRNA-mediated gene silencing: a look under the hood of miRISC. Nat Struct Mol Biol. 2012; 19(6): 586-93.

9. Carstens JL, Lovisa S, Kalluri R. Microenvironment-dependent cues trigger miRNA-regulated feedback loop to facilitate the EMT/MET switch. Journal of Clinical Investigation. 2014; 124(4): 1458.

10. Kandhavelu J, Subramanian K, Khan A, Omar A, Ruff P, Penny C. Computational Analysis of miRNA and their Gene Targets Significantly Involved in Colorectal Cancer Progression. MicroRNA. 2019; 8(1): 68-75.

11. Usuba W, Urabe F, Yamamoto Y, Matsuzaki J, Sasaki H, Ichikawa M, et al. Circulating miRNA panels for specific and early detection in bladder cancer. Cancer science. 2019; 110(1): 408-19.

12. Wang X, An D, Liu X, Wang X, Li B. MicroRNA-27a downregulates the expression of Hsp90 and enhances the radiosensitivity in esophageal squamous cell carcinoma. OncoTargets and therapy. 2019; 12: 5967-77.

13. El Bezawy R, Tinelli S, Tortoreto M, Doldi V, Zuco V, Folini M, et al. miR-205 enhances radiation sensitivity of prostate cancer cells by impairing DNA damage repair through PKCepsilon and ZEB1 inhibition. Journal of experimental \& clinical cancer research. 2019; 38(1): 51.

14. Yue X, Lan F, Xia T. Hypoxic Glioma Cell-Secreted Exosomal miR-301a Activates Wnt/ $\beta$-catenin Signaling and Promotes Radiation Resistance by Targeting TCEAL7. Molecular therapy. 2019; 27(11): 1939-49.

15. Guo Y, Zhang Y, Zhang SJ, Ma YN, He Y. Comprehensive analysis of key genes and microRNAs in radioresistant nasopharyngeal carcinoma. BMC medical genomics. 2019; 12(1): 73.

16. Li G, Qiu Y, Su Z, Ren S, Liu C, Tian Y, et al. Genome-wide analyses of radioresistance-associated miRNA expression profile in nasopharyngeal carcinoma using next generation deep sequencing. PloS one. 2013; 8(12): e84486.

17. Bao B, Li Y, Ahmad A, Azmi AS, Bao G, Ali S, et al. Targeting CSC-Related miRNAs for Cancer Therapy by Natural Agents. Current drug targets. 2012; 13(14): 1858-68.

18. Barker HE, Paget JTE, Khan AA, Harrington KJ. The Tumour Microenvironment after Radiotherapy: Mechanisms of Resistance and Recurrence. Nature reviews Cancer. 2015; 15(7): 409-25.

19. Lv Y, Yang H, Ma X, Wu G. Strand-specific miR-28-3p and miR-28-5p have differential effects on nasopharyngeal cancer cells proliferation, apoptosis, migration and invasion. Cancer cell international. 2019; 19: 187.

20. Lan F, Qin Q, Yu H, Yue X. Effect of glycolysis inhibition by miR-448 on glioma radiosensitivity. Journal of neurosurgery. 2019: 1-9.

21. Zhang T, Xue X, Peng H. Therapeutic Delivery of miR-29b Enhances Radiosensitivity in Cervical Cancer. Molecular therapy. 2019; 27(6): 1183-94.

22. Macklis RM, Lin JY, Beresford B, Atcher RW, Hines JJ, Humm JL. Cellular kinetics, dosimetry, and radiobiology of alpha-particle radioimmunotherapy: induction of apoptosis. Radiation research. 1992; 130(2): $220-6$
23. Cheng J, He S, Wang M, Zhou L, Zhang Z, Feng $X$, et al. The Caspase-3/PKCס/Akt/VEGF-A Signaling Pathway Mediates Tumor Repopulation during Radiotherapy. Clinical cancer research. 2019; 25(12): 3732-43

24. de Almeida VH, de Melo AC, Meira DD, Pires AC, Nogueira-Rodrigues A, Pimenta-Inada $\mathrm{HK}$, et al. Radiotherapy modulates expression of EGFR, ERCC1 and p53 in cervical cancer. Brazilian Journal of Medical and Biological Research. 2018; 51(1): e6822.

25. Zhang G, Wang W, Yao C, Zhang S, Liang L, Han M, et al. Radiation-resistant cancer stem-like cell properties are regulated by PTEN through the activity of nuclear $\beta$-catenin in nasopharyngeal carcinoma. Oncotarget. 2017; 8(43): 74661-72.

26. Aggarwal BB, Sung B. NF-кB in cancer: a matter of life and death. Cancer Discovery. 2011; 1(6): 469

27. Lee JM, Bernstein A. p53 mutations increase resistance to ionizing radiation. Proc Natl Acad Sci U S A. 1993; 90(12): 5742-6.

28. Farideh H, Reza SM, Nasrollah J, Hossein M. Modulating Survivin as a Radioresistant Factor, Caspase-3, and Apoptosis by Omega-3 Docosahexaenoic Acid Sensitizes Mutant-p53 Colorectal Cancer Cells to Y-Irradiation. Cancer Biotherapy and Radiopharmaceuticals. 2018; 33(9): 387-95.

29. Zheng Z, Qu J-Q, Yi H-M, Ye X, Huang W, Xiao T, et al. MiR-125b regulates proliferation and apoptosis of nasopharyngeal carcinoma by targeting A20/NF-kB signaling pathway. Cell Death \& Disease. 2017; 8(6): e2855.

30. Savic V, Yin B, Maas NL, Bredemeyer AL, Carpenter AC, Helmink BA, et al. Formation of dynamic gamma-H2AX domains along broken DNA strands is distinctly regulated by ATM and MDC1 and dependent upon H2AX densities in chromatin. Molecular cell. 2009; 34(3): 298-310.

31. Lung RW, Hau PM, Yu KH, Yip KY, Tong JH, Chak WP, et al. EBV-encoded miRNAs target ATM-mediated response in nasopharyngeal carcinoma. The Journal of pathology. 2018; 244(4): 394-407.

32. Bruce JP, Liu F-F. MicroRNAs in nasopharyngeal carcinoma. Chin J Cancer. 2014; 33(11): 539-44.

33. Liu M, Zhu K, Qian X, Li W. Identification of miRNA/mRNA-Negative Regulation Pairs in Nasopharyngeal Carcinoma. Med Sci Monit. 2016; 22: $2215-34$

34. Wang $\mathrm{Y}$, Zhao Q, Lan N, Wang S. Identification of methylated genes and miRNA signatures in nasopharyngeal carcinoma by bioinformatics analysis. Mol Med Rep. 2018; 17(4): 4909-16.

35. Lee KT-W, Tan J-K, Lam AK-Y, Gan S-Y. MicroRNAs serving as potential biomarkers and therapeutic targets in nasopharyngeal carcinoma: A critical review. Crit Rev Oncol Hematol. 2016; 103: 1-9.

36. Gu L, Shi Y, Xu W, Ji Y. PPAR $\beta / \delta$ Agonist GW501516 Inhibits Tumorigenesis and Promotes Apoptosis of the Undifferentiated Nasopharyngeal Carcinoma C666-1 Cells by Regulating miR-206. Oncol Res. 2019; 27(8): 923-33.

37. Xu Y-J, Zhou R, Zong J-F, Lin W-S, Tong S, Guo Q-J, et al. Epstein-Barr virus-coded miR-BART13 promotes nasopharyngeal carcinoma cell growth and metastasis via targeting of the NKIRAS2/NF-kB pathway. Cancer Lett. 2019; 447: 33-40.

38. Zou X, Zhu D, Zhang H, Zhang S, Zhou X, He X, et al. MicroRNA expression profiling analysis in serum for nasopharyngeal carcinoma diagnosis. Gene. 2020; 727: 144243.

39. Peng J, Feng Y, Rinaldi G, Levine P, Easley S, Martinez E, et al. Profiling miRNAs in nasopharyngeal carcinoma FFPE tissue by microarray and Next Generation Sequencing. Genomics data. 2014; 2: 285-9.

40. Li X-H, Ou J-O, Yi H, Zhang P-F, Yi H-M, Wan X-X, et al. Integrated Analysis of Differential miRNA and mRNA Expression Profiles in Human Radioresistant and Radiosensitive Nasopharyngeal Carcinoma Cells. PloS one. 2014; 9(1): e87767.

41. Qu C, Liang Z, Huang J, Zhao R, Su C, Wang S, et al. MiR-205 determines the radioresistance of human nasopharyngeal carcinoma by directly targeting PTEN. Cell cycle. 2012; 11(4): 785-96.

42. Mao Y, Wu S, Zhao R, Deng Q. MiR-205 promotes proliferation, migration and invasion of nasopharyngeal carcinoma cells by activation of AKT signalling. The Journal of International Medical Research. 2016; 44(2): 231-40.

43. Huang D, Bian G, Pan Y, Han X, Sun Y, Wang Y, et al. MiR-20a-5p promotes radio-resistance by targeting Rab27B in nasopharyngeal cancer cells. Cancer cell international. 2017; 17: 32

44. Zhao F, Pu Y, Qian L, Zang C, Tao Z, Gao J. MiR-20a-5p promotes radio-resistance by targeting NPAS2 in nasopharyngeal cancer cells. Oncotarget. 2017; 8(62): 105873-81.

45. Kong L, Wei Q, Hu X, Chen L, Li J. miR-193a-3p Promotes Radio-Resistance of Nasopharyngeal Cancer Cells by Targeting SRSF2 Gene and Hypoxia Signaling Pathway. Medical science monitor basic research. 2019; 25: 53-62. 
46. Edison N, Curtz Y, Paland N, Mamriev D, Chorubczyk N, Haviv-Reingewertz T, et al. Degradation of Bcl-2 by XIAP and ARTS Promotes Apoptosis. Cell reports. 2017; 21(2): 442-54.

47. Cory S, Adams JM. The Bcl2 family: regulators of the cellular life-or-death switch. Nature reviews Cancer. 2002; 2(9): 647-56.

48. Wang W, Mani AM, Wu Z-H. DNA damage-induced nuclear factor-kappa B activation and its roles in cancer progression. Journal of cancer metastasis and treatment. 2017; 3: 45-59.

49. Ou H, Li Y, Kang M. Activation of miR-21 by STAT3 induces proliferation and suppresses apoptosis in nasopharyngeal carcinoma by targeting PTEN gene. PloS one. 2014; 9(11): e109929.

50. Dai B, Meng J, Peyton M, Girard L, Bornmann WG, Ji L, et al. STAT3 mediates resistance to MEK inhibitor through microRNA miR-17. Cancer research. 2011; 71(10): 3658-68.

51. Li L-N, Xiao T, Yi H-M, Zheng Z, Qu J-Q, Huang W, et al. MiR-125b Increases Nasopharyngeal Carcinoma Radioresistance By Targeting A20/NF-kB Signaling Pathway. Molecular cancer therapeutics. 2017; 16(10): 2094-2106.

52. Cheng JZ, Chen JJ, Wang ZG, Yu D. MicroRNA-185 inhibits cell proliferation while promoting apoptosis and autophagy through negative regulation of TGF-beta1/mTOR axis and HOXC6 in nasopharyngeal carcinoma. Cancer biomarkers. 2018; 23(1): 107-23.

53. Wu Q, Han T, Sheng X, Zhang N, Wang P. Downregulation of EB virus miR-BART4 inhibits proliferation and aggressiveness while promoting radiosensitivity of nasopharyngeal carcinoma. Biomedicine \& pharmacotherapy. 2018; 108: 741-51.

54. Chen JH, Yang R, Zhang W, Wang YP. Functions of microRNA-143 in the apoptosis, invasion and migration of nasopharyngeal carcinoma. Experimental and therapeutic medicine. 2016; 12(6): 3749-55.

55. Guo G, Zhang Y, Hu L, Bian X. MicroRNA-153 affects nasopharyngeal cancer cell viability by targeting TGF-beta2. Oncology letters. 2019; 17(1): 646-51.

56. Xu F, Ren L, Song M, Shao B, Han Y, Cao Z, et al. Fas- and Mitochondria-Mediated Signaling Pathway Involved in Osteoblast Apoptosis Induced by AlCl3. Biological trace element research. 2018; 184(1): 173-85.

57. Poreba M, Groborz K, Navarro M, Snipas SJ, Drag M, Salvesen GS. Caspase selective reagents for diagnosing apoptotic mechanisms. Cell Death \& Differentiation. 2019; 26(2): 229-44.

58. Sang Eun H, Seong Min K, Ho Jeong L, Vetrivel P, Venkatarame Gowda Saralamma V, Jeong Doo $\mathrm{H}$, et al. Scutellarein Induces Fas-Mediated Extrinsic Apoptosis and G2/M Cell Cycle Arrest in Hep3B Hepatocellular Carcinoma Cells. Nutrients. 2019; 11(2): 263.

59. Chan ATC, Hui EP, Ngan RKC, Tung SY, Cheng ACK, Ng WT, et al. Analysis of Plasma Epstein-Barr Virus DNA in Nasopharyngeal Cancer After Chemoradiation to Identify High-Risk Patients for Adjuvant Chemotherapy: A Randomized Controlled Trial. Journal of clinical oncology. 2018: Jco2018777847.

60. Yang G-D, Huang T-J, Peng L-X, Yang C-F, Liu R-Y, Huang H-B, et al. Epstein-Barr Virus_Encoded LMP1 upregulates microRNA-21 to promote the resistance of nasopharyngeal carcinoma cells to cisplatin-induced Apoptosis by suppressing PDCD4 and Fas-L. PloS one. 2013; 8(10): e78355-e.

61. Yu Y, Liang H, Lv X, Ke L, Qiu W, Huang X, et al. Platinum-based concurrent chemotherapy remains the optimal regimen for nasopharyngeal carcinoma: a large institutional-based cohort study from an endemic area. Journal of cancer research and clinical oncology. 2018; 144(11): 2231-43.

62. Pastor M, Lopez Pousa A, Del Barco E, Perez Segura P, Astorga BG, Castelo B, et al. SEOM clinical guideline in nasopharynx cancer (2017). Clinical \& translational oncology. 2018; 20(1): 84-8.

63. Zhou X, Zheng J, Tang Y, Lin Y, Wang L, Li Y, et al. EBV encoded miRNA BART8-3p promotes radioresistance in nasopharyngeal carcinoma by regulating ATM/ATR signaling pathway. Bioscience reports. 2019; 39(9): BSR20190415.

64. Su M, Wang H, Wang W, Wang Y, Ouyang L, Pan C, et al. LncRNAs in DNA damage response and repair in cancer cells. Acta biochimica et biophysica Sinica. 2018; 50(5): 433-9.

65. Kang M, Xiao J, Wang J, Zhou P, Wei T, Zhao T, et al. MiR-24 enhances radiosensitivity in nasopharyngeal carcinoma by targeting SP1. Cancer medicine. 2016; 5(6): 1163-73.

66. Wang S, Pan Y, Zhang R, Xu T, Wu W, Zhang R, et al. Hsa-miR-24-3p increases nasopharyngeal carcinoma radiosensitivity by targeting both the 3'UTR and 5'UTR of Jab1/CSN5. Oncogene. 2016; 35(47): 6096-108.

67. Doronkin S, Djagaeva I, Beckendorf SK. CSN5/Jab1 mutations affect axis formation in the Drosophila oocyte by activating a meiotic checkpoint. Development. 2002; 129(21): 5053-64.
68. Pan Y, Zhang Q, Tian L, Wang X, Fan X, Zhang H, et al. Jab1/CSN5 negatively regulates p27 and plays a role in the pathogenesis of nasopharyngeal carcinoma. Cancer Res. 2012; 72(7): 1890-900.

69. Pan Y, Zhang Q, Atsaves V, Yang H, Claret FX. Suppression of Jab1/CSN5 induces radio- and chemo-sensitivity in nasopharyngeal carcinoma through changes to the DNA damage and repair pathways. Oncogene. 2013; 32(22): 2756-66.

70. Ciccia A, Elledge SJ. The DNA damage response: making it safe to play with knives. Molecular cell. 2010; 40(2): 179-204.

71. Nie X, Guo E, Wu C, Liu D, Sun W, Zhang L, et al. SALL4 induces radioresistance in nasopharyngeal carcinoma via the $\mathrm{ATM} / \mathrm{Chk} 2 / \mathrm{p} 53$ pathway. Cancer medicine. 2019; 8(4): 1779-92.

72. Bartek J, Lukas J. DNA damage checkpoints: from initiation to recovery or adaptation. Current Opinion in Cell Biology. 2007; 19(2): 238-45.

73. Hashimoto T, Murata Y, Urushihara Y, Shiga S, Takeda K, Hosoi Y, Severe hypoxia increases expression of ATM and DNA-PKCs and it increases their activities through Src and AMPK signaling pathways. Biochemical and biophysical research communications. 2018; 505(1): 13-9.

74. Giacinti C, Giordano A. RB and cell cycle progression. Oncogene. 2006; 25(38): 5220-7.

75. Wu J, Lv Q, He J, Zhang H, Mei X, Cui K, et al. MicroRNA-188 suppresses G1/S transition by targeting multiple cyclin/CDK complexes. Cell communication and signaling. 2014; 12: 66 .

76. Lu J, He M-L, Wang L, Chen Y, Liu X, Dong Q, et al. MiR-26a inhibits cell growth and tumorigenesis of nasopharyngeal carcinoma through repression of EZH2. Cancer research. 2011; 71(1): 225-33.

77. Qu JQ, Yi HM, Ye X, Li LN, Zhu JF, Xiao T, et al. MiR-23a sensitizes nasopharyngeal carcinoma to irradiation by targeting IL-8/Stat3 pathway. Oncotarget. 2015; 6(29): 28341-56.

78. Liang S, Zhang N, Deng Y, Chen L, Zhang Y, Zheng Z, et al. miR-663 promotes NPC cell proliferation by directly targeting CDKN2A. Mol Med Rep. 2017; 16(4): 4863-70.

79. McKelvey KJ, Hudson AL, Back M, Eade T, Diakos CI. Radiation, inflammation and the immune response in cancer. Mammalian genome. 2018; 29(11-12): 843-65.

80. Narayanan PK, LaRue KE, Goodwin EH, Lehnert BE. Alpha particles induce the production of interleukin- 8 by human cells. Radiation research. 1999; 152(1): 57-63.

81. Qu JQ, Yi HM, Ye X, Zhu JF, Yi H, Li LN, et al. MiRNA-203 Reduces Nasopharyngeal Carcinoma Radioresistance by Targeting IL8/AKT Signaling. Mol Cancer Ther. 2015; 14(11): 2653-64

82. Wang LX, Kang ZP, Yang ZC, Ma RX, Tan Y, Peng XB, et al. MicroRNA-135a Inhibits Nasopharyngeal Carcinoma Cell Proliferation Through Targeting Interleukin-17. Cellular physiology and biochemistry. 2018; 46(6): 2232-8.

83. Matsuoka Y, Nakayama H, Yoshida R, Hirosue A, Nagata M, Tanaka T, et al. IL- 6 controls resistance to radiation by suppressing oxidative stress via the Nrf2-antioxidant pathway in oral squamous cell carcinoma. British journal of cancer. 2016; 115(10): 1234-44.

84. Batlle E, Clevers H. Cancer stem cells revisited. Nat Med. 2017; 23(10): 1124-34.

85. Cai L, Long $\mathrm{Y}$, Chong $\mathrm{T}$, Cai W, Tsang CM, Zhou X, et al. EBV-miR-BART7-3p Imposes Stemness in Nasopharyngeal Carcinoma Cells by Suppressing SMAD7. Front Genet. 2019; 10: 939.

86. Wang W, Wu S, Shi Y, Miao Y, Luo X, Ji M, et al. c-MYB regulates cell growth and DNA damage repair through modulating MiR-143. FEBS Lett. 2015; 589(5): 555-64.

87. Jiang $\mathrm{O}$, Zhou $\mathrm{Y}$, Yang $\mathrm{H}$, Li L, Deng $\mathrm{X}$, Cheng $\mathrm{C}$, et al. A directly negative interaction of miR-203 and ZEB2 modulates tumor stemness and chemotherapy resistance in nasopharyngeal carcinoma. Oncotarget. 2016; 7(41): 67288-301.

88. Zhang G, Zong J, Lin S, Verhoeven RJA, Tong S, Chen Y, et al. Circulating Epstein-Barr virus microRNAs miR-BART7 and miR-BART13 as biomarkers for nasopharyngeal carcinoma diagnosis and treatment. Int J Cancer. 2015; 136(5): E301-E12.

89. Liu B, Tan Z, Jiang Y, Chen Y, Chen Y, Ling K. Correlation between the expression of miR150 and FOXO4 and the local recurrence and metastasis of nasopharyngeal carcinoma after intensive radiotherapy. J BUON. 2018; 23(6): 1671-8.

90. Zhang H, Zou X, Wu L, Zhang S, Wang T, Liu P, et al. Identification of a 7-microRNA signature in plasma as promising biomarker for nasopharyngeal carcinoma detection. Cancer medicine. 2020; 9(3): 1230-41.

91. Vogt PK, Gymnopoulos M, Hart JR. PI 3-kinase and cancer: changing accents. Current Opinion in Genetics \& Development. 2009; 19(1): 12.

92. Li G, Liu Y, Su Z, Ren S, Zhu G, Tian Y, et al. MicroRNA-324-3p regulates nasopharyngeal carcinoma radioresistance by directly targeting WNT2B. European journal of cancer. 2013; 49(11): 2596-607. 
93. Huang Y, Tan D, Xiao J, Li Q, Zhang X, Luo Z. miR-150 contributes to the radioresistance in nasopharyngeal carcinoma cells by targeting glycogen synthase kinase-3beta. Journal of cancer research and therapeutics. 2018; 14(1): 111-8.

94. Wu W, Chen X, Yu S, Wang R, Zhao R, Du C. microRNA-222 promotes tumor growth and confers radioresistance in nasopharyngeal carcinoma by targeting PTEN. Mol Med Rep. 2018; 17(1): 1305-10.

95. Feng $\mathrm{X}, \mathrm{Lv} \mathrm{W}$, Wang $\mathrm{S}, \mathrm{He} \mathrm{Q}$. miR495 enhances the efficacy of radiotherapy by targeting GRP78 to regulate EMT in nasopharyngeal carcinoma cells. Oncology reports. 2018; 40(3): 1223-32.

96. Wang S, Claret FX, Wu W. MicroRNAs as Therapeutic Targets in Nasopharyngeal Carcinoma. Frontiers in oncology. 2019; 9: 756.

97. Tan LP, Tan GW, Sivanesan VM, Goh SL, Ng XJ, Lim CS, et al. Systematic comparison of plasma EBV DNA, anti-EBV antibodies and miRNA levels for early detection and prognosis of nasopharyngeal carcinoma. Int J Cancer. 2020; 146 (8), 2336-2347. 